\title{
A RATIO TEST
}

\section{ORRIN FRINK}

The d'Alembert ratio test has numerous extensions that are effective in some cases where the d'Alembert test fails; that is, when the limit of the ratio $a_{n} / a_{n-1}$ is 1 . Examples are Raabe's test and Gauss' test. The following test is simpler than any of these, and is easy to prove and to remember.

Theorem. A series of positive terms (1) $\sum a_{n}$ converges if $\lim _{n \rightarrow \infty}\left(a_{n} / a_{n-1}\right)^{n}<1 / e$, and diverges if $\lim _{n \rightarrow \infty}\left(a_{n} / a_{n-1}\right)^{n}>1 / e$.

More generally, (1) converges if $\lim \sup \left(a_{n} / a_{n-1}\right)^{n}<1 / e$. It diverges if $\left(a_{n} / a_{n-1}\right)^{n} \geqq 1 / e$ for all $n$ sufficiently large. In particular (1) diverges if $\lim \inf \left(a_{n} / a_{n-1}\right)^{n}>1 / e$.

Corollary. A series of real or complex terms $\sum a_{n}$ converges absolutely if lim sup $\left|a_{n} / a_{n-1}\right|^{n}<1 / e$.

The proof is by the comparison ratio test with $\sum n^{-8}$ as the comparison series. To prove the convergence part of the test, suppose $\lim \sup \left(a_{n} / a_{n-1}\right)^{n}=e^{-d}<e^{-1}$. Then $d>1$. Consider the series (2) $\sum b_{n}=\sum n^{-s}$ with $1<s<d$. Then we have $\lim \left(b_{n} / b_{n-1}\right)^{n}$ $=\lim (1-1 / n)^{n s}=e^{-s}$. Since $e^{-d}<e^{-s}$, then ultimately $\left(a_{n} / a_{n-1}\right)^{n}$ $<\left(b_{n} / b_{n-1}\right)^{n}$, and therefore $a_{n} / a_{n-1}<b_{n} / b_{n-1}$. It follows that (1) converges, since (2) does.

To prove the divergence part of the test, suppose $\left(a_{n} / a_{n-1}\right)^{n} \geqq 1 / e$ for $n$ sufficiently large. For the harmonic series (3) $\sum c_{n}=\sum n^{-1}$, we have $\left(c_{n} / c_{n-1}\right)^{n}=(1-1 / n)^{n}<e^{-1}$ for all $n$. Hence ultimately $\left(a_{n} / a_{n-1}\right)^{n}$ $>\left(c_{n} / c_{n-1}\right)^{n}$, and therefore $a_{n} / a_{n-1}>c_{n} / c_{n-1}$. Since (3) diverges, so does (1). This completes the proof. In the same manner one can prove the following generalization.

Theorem. A series of positive terms (1) $\sum a_{n}$ converges if $\lim \sup \left(a_{n} / a_{n-k}\right)^{n}<1 / e^{k}$, and diverges if $\left(a_{n} / a_{n-k}\right)^{n} \geqq 1 / e^{k}$ for all $n$ sufficiently large.

As an application, consider the Dirichlet series (4) $\sum a_{n} n^{-s}$, where $s=\sigma+i \tau$. Suppose $\lim \left|a_{n} / a_{n-1}\right|^{n}=e^{-d}$. Then it follows from the test that the series (4) converges absolutely at every point of the halfplane $\sigma>1-d$, and converges absolutely at no point of the halfplane $\sigma<1-d$.

\section{The Pennsylvania State College}

Presented to the Society, April 17, 1948; received by the editors December 24, 1947. 